\title{
Conformal symmetry limit of QED and QCD and identities between perturbative contributions to deep-inelastic scattering sum rules
}

\author{
A.L. Kataev \\ Institute for Nuclear Resrach of the Russian Academy of Sciences, \\ 60th October Anniversary Prospect 7a, 117312 Moscow, Russia \\ E-mail: kataev@ms2.inr.ac.ru
}

ABSTRACT: Conformal symmetry-based relations between concrete perturbative QED and QCD approximations for the Bjorken, the Ellis-Jaffe sum rules of polarized lepton- nucleon deep-inelastic scattering (DIS), the Gross-Llewellyn Smith sum rules of neutrino-nucleon DIS, and for the Adler functions of axial-vector and vector channels are derived. They result from the application of the operator product expansion to three triangle Green functions, constructed from the non-singlet axial-vector, and two vector currents, the singlet axialvector and two non-singlet vector currents and the non-singlet axial-vector, vector and singlet vector currents in the limit, when the conformal symmetry of the gauge models with fermions is considered unbroken. We specify the perturbative conditions for this symmetry to be valid in the case of the $\mathrm{U}(1)$ and $\mathrm{SU}\left(N_{c}\right)$ models. The all-order perturbative identity following from the conformal invariant limit between the concrete contributions to the Bjorken, the Ellis-Jaffe and the Gross-Llewellyn Smith sum rules is proved. The analytical and numerical $O\left(\alpha^{4}\right)$ and $O\left(\alpha_{s}^{2}\right)$ conformal symmetry based approximations for these sum rules and for the Adler function of the non-singlet vector currents are summarized. Possible theoretical applications of the results presented are discussed.

KeYwords: Deep Inelastic Scattering, Conformal and W Symmetry

ARXiv EPRINT: 1305.4605 


\section{Contents}

1 Introduction 1

2 The AVV three-point functions and the conformal symmetry in the U(1) and $\mathrm{SU}\left(N_{c}\right)$ models

2.1 Basic considerations

2.2 The definitions of the perturbative coefficient functions

$\begin{array}{lll}2.3 & \text { The definitions of the } \mathrm{SU}\left(N_{c}\right) \text { and } \mathrm{U}(1) \text { group weights } & 7\end{array}$

2.4 Specification of the conformally invariant limit in perturbative QED 9

3 Conformal symmetry governed contributions to the coefficient functions of the DIS sum rules in QED and QCD

3.1 The all order perturbative identities between coefficient functions of DIS sum rules in the conformal symmetry limit

3.2 Concrete analytical and numerical results

\section{Introduction}

The concepts of scale-invariance and of conformal symmetry (CS) play an important role in studies of perturbative approximations for various three-point and two-point Green functions of the renormalized massless quantum gauge models with fermions. It was proved in the coordinate space-time representation, that in the CS limit of these models, which is realized when coupling constants are considered as non-renormalized bare parameters (i.e. when $\alpha=\alpha^{B}$ or $\alpha_{s}=\alpha_{s}^{B}$ ), the multiloop expression for the AVV three-point Green function of the flavour non-singlet (NS) axial-vector and two vector currents coincides with the lowest-order 1-loop triangle graph [1]. In the momentum space-time representation the result of ref. [1] was rewritten in ref. [2] as

$$
T_{\mu \alpha \beta}^{c a b}(p, q)=i \int<0\left|T A_{\mu}^{c}(y) V_{\alpha}^{a}(x) V_{\beta}^{b}(0)\right| 0>e^{i p x+i q y} d x d y=d_{R}^{c a b} \Delta_{\mu \alpha \beta}^{1-l}(p, q)
$$

Here $A_{\mu}^{c}(y)=\bar{\psi}(y) \gamma_{\mu}\left(\lambda^{c} / 2\right) \gamma_{5} \psi(y)$ and $V_{\alpha}^{a}(x)=\bar{\psi}(x) \gamma_{\alpha}\left(\lambda^{a} / 2\right) \psi(x)$ are the NS axial-vector and vector currents, $d_{R}^{c a b}$ is the symmetric structure constant of the $\mathrm{SU}\left(N_{c}\right)$ group with its generators $\left(\lambda^{a} / 2\right),\left(\lambda^{b} / 2\right),\left(\lambda^{c} / 2\right)$ defined in the representation $R$ of the related Lie algebra and $\Delta_{\mu \alpha \beta}^{1-l}(p, q)$ is the 1-loop contribution to the triangle Green function. In ref. [3] the validity of this non-renormalization property was explicitly demonstrated at the 2loop level using differential regularization and differential renormalization prescriptions, proposed in ref. [4]. For the dimensional regularization [5-9] and in the $\overline{M S}$-scheme [10], 
formulated in more detail in [11], the cancellation of all 2-loop internal contributions to the AVV three-point function were rediscovered in ref. [12]. The agreement of this result with the outcomes of 2-loop calculations, performed in ref. [3] using differential regularization and renormalization approaches, is not accidental. Indeed, the differential renormalization can be straightforwardly related to the dimensional regularization and the $\overline{M S}$-scheme [13].

In the present work we consider two extra AVV three-point Green functions, which are closely related to the one of eq. (1.1). The first of them is constructed from the singlet (SI) axial-vector and two NS vector currents, namely

$$
\tilde{T}_{\mu \alpha \beta}^{a b}(p, q)=i \int<0\left|T A_{\mu}(y) V_{\alpha}^{a}(x) V_{\beta}^{b}(0)\right| 0>e^{i p x+i q y} d x d y .
$$

where $A_{\mu}(y)=\bar{\psi}(y) \gamma_{\mu} \gamma_{5} \psi(y)$ is the SI axial-vector fermion current. The properties of this Green function were investigated previously in ref. [14] within the deeply investigated finite QED program (see e.g. refs. $[15,16]$ ). This program had the aim to find out whether a nontrivial ultraviolet zero may exist in the RG $\beta$-function of the perturbative quenched QED (pqQED) model ( which will be defined in section 2) or in the QED Gell-Mann-Low function $\Psi(\alpha)$ [17], which as clarified in the review of ref. [18] is identical to the QED $\beta$-function in the momentum subtractions scheme (see e.g. ref. [19] for the detailed explanation ). Using the methods of the finite QED program, it was shown in ref. [16], that if an ultraviolet zero of $\Psi(\alpha)$ exists, it should be a zero of infinite order. Now we know that this feature is not realized in perturbative QED. However, the important understanding of the basic features of the methods and the symmetries, gained in the process of investigations of the of the finite QED program, remain important today. Among them is the notion of the CS limit of QED. The consequences of the possible applications of the limit to the perturbative expression of eq. (1.2) were not considered in ref. [14]. The concrete analytical high-order perturbative results, which follow from this limit, were obtained only recently [20].

The second three-point Green function we will be interested in is constructed from the NS axial-vector, the NS vector and SI vector fermion currents with $N_{F}$ number of fermions, namely

$$
\tilde{\tilde{T}}_{\mu \alpha \beta}^{a b}(p, q)=i \int<0\left|T A_{\mu}^{a}(y) V_{\alpha}^{b}(0) V_{\beta}(y)\right| 0>e^{i q x-i p y} d x d y \quad .
$$

Here $V_{\beta}(0)=N_{F} \bar{\psi}_{i}(0) \gamma_{\alpha} \psi_{i}(0)$ is the SI vector quark current with $N_{F}$ fermions of unit charge. Theoretical consequences, which will be obtained from these two AVV functions of eq. (1.2) and eq. (1.3) are new and were not published in the regular journal, though some of them were already discussed by the author (see [21], [22]).

The most important result, derived from eq. (1.1) in the conformally invariant limit, is the relation between $\pi \rightarrow \gamma \gamma$ decay constant and the product of the Bjorken sum rule of the polarized deep-inelastic scattering (DIS) and the Adler functions, related to the total cross-section of the $e^{+} e^{-}$-annihilation to hadrons process, both evaluated in the Born approximation [23]. Other relations, which follow from the application of the operator product expansion (OPE) approach to the same AVV Green function in different kinematic regimes, were derived in ref. [24]. In this work the basic Crewther relation of ref. [23] was generalized to the the level of the $O\left(\alpha^{2}\right)$ corrections within finite QED program. 
In the case of the $\mathrm{SU}\left(N_{c}\right)$ gauge model with fermions the most important understanding of the properties of the perturbative series in the case when the CS exist and when it is violated by the $\overline{M S}$ renormalization procedure was achieved in ref. [25]. In this work the generalized QCD Crewther relation between the analytical $\overline{M S}$-scheme $O\left(\alpha_{s}^{3}\right)$ approximations for the Bjorken polarized (Bjp) sum rule, the Gross-Llewellyn Smith (GLS) sum rule [26] and the similar approximation for the $e^{+} e^{-}$-annihilation Adler Function, evaluated in refs. $[27,28]^{1}$ and independently confirmed in ref. [30], was discovered. The factorization of the the 2-loop $\mathrm{RG} \beta$-function of the $\mathrm{SU}\left(N_{c}\right)$-model was revealed in the generalized $\overline{M S}$-scheme Crewther relation at the $\alpha_{s}^{3}$-level.

The analytical calculations of the $\alpha_{s}^{4}$-corrections to the Bjp sum rule [31], the GLS sum rule, the $D$-function in the NS and vector channels allowed the authors of refs. [31, $32]$ to demonstrate explicitly the existence of the closed $\overline{M S}$-scheme approximations for the generalized QCD Crewther relations [25] with the factorized 3-loop RG $\beta$-function, analytically evaluated in ref. [33] and confirmed in ref. [34]. The validity of the $\overline{M S}$ scheme relation for the Bjp sum rule to all orders of perturbation theory was considered in momentum space [2] and then proved in the coordinate space-time representation $[35,36]$ without specifying the form of high order perturbative QCD corrections.

The additional SI-type $\alpha_{s}^{4}$ contributions to the $O\left(\alpha_{s}^{4}\right)$ NS expression for the Bjp sum rule [31] were obtained recently $[37]^{2}$ from the equality of the generalizations of the Crewther relations for the product of the coefficient function of the Bjp sum rule and the NS $D$-function and the product of the coefficient functions of the GLS sum rule and the $D$-function of vector currents. This equality was proposed in ref. [21] and published in ref. [38] without taking into account SI-type $O\left(\alpha_{s}^{4}\right)$-corrections to the Bjp sum rule. Note, that the Crewther relation for the GLS sum rule was analysed in the Born QED approximation [24], in the $O\left(a_{s}^{3}\right)$ and $O\left(a_{s}^{4}\right) \mathrm{SU}\left(N_{c}\right)$ studies of ref. [25] and ref. [32], and in the general theoretical perturbative QCD analysis of refs. [35, 36] as well.

In this work we derive new perturbative all-order identities between the concrete approximations for the coefficient functions of the Bjp, Ellis-Jaffe (EJ) and the GLS sum rules. In the CS limit, which can be formulated for QED and hopefully for QCD, these approximations should be related to similar perturbative expressions for the $D$-functions of the NS and SI vector currents. We discuss how this CS limit of U(1) gauge model can be specified within perturbation theory. It is stressed, that the origin of this limit differs from a similar limit, considered in the works which are devoted to the searches for fixed points or "conformally invariant windows" in the expressions for the RG $\beta$-functions of the gauge model under consideration (see e.g. refs. [39, 40]). Its realization also differs from the proposal of ref. [41] to restore the CS of renormalized QED by modification of its Lagrangian in an arbitrary number of dimensions. In our case the mechanism which realizes the CS is simulating in part the one that responsible for the CS in $\mathcal{N}=4 \mathrm{SYM}$ theory with an identical zero RG $\beta$-function.

Theoretical applications of the results obtained, including the explanation of the cancellations between SI-type perturbative $\alpha_{s}^{3}$ contributions to the GLS sum rule and to the

\footnotetext{
${ }^{1}$ In numerical form the result of ref. [27] was published in ref. [29].

${ }^{2}$ The results are not yet verified by direct analytical calculations.
} 
Adler $D$-function of fermion vector currents [25] and of the specific SI $\alpha_{s}^{4}$ corrections to both quantities [32] are presented. We consider also the structure of the available U(1) perturbative expansions for the DIS sum rule and for the Adler functions. The application of the certain $\alpha_{s}^{2} \mathrm{SU}\left(N_{c}\right)$ results for these quantities, which follow from this definition of the conformally-invariant limit, are discussed.

\section{The AVV three-point functions and the conformal symmetry in the $\mathrm{U}(1)$ and $\mathrm{SU}\left(N_{c}\right)$ models}

\subsection{Basic considerations}

Let us study the three-point functions of eq. (1.2) and eq. (1.3) in the conformally invariant limits of the $\mathrm{U}(1)$ and $S U\left(N_{c}\right)$ gauge models with fermions. They are realized when the coupling constants and gauge models and the external SI vector currents $V_{\mu}$ in eq. (1.3) are fixed as the bare unrenormalized quantities. In other words, in the expressions of the AVV three-point functions of eq. (1.2) and eq. (1.3) the coupling constants and the concrete currents are fixed as $\alpha=\alpha^{B}, \alpha_{s}=\alpha_{s}^{B}$ and $A_{\mu}=A_{\mu}^{B}(x), V_{\mu}(x)=V_{\mu}^{B}(x)$.

In this case two NS vector currents in the three-point Green function of eq. (1.2) and the SI vector current in the three-point Green function of eq. (1.3) are conserved by definition, while the SI axial-vector operator $A_{\mu}^{B}(x)$ in the three-point Green function of eq. (1.2) and the SI vector operator $V_{\mu}^{B}(x)$ are not renormalized by construction (we recall that the bare operators do not depend on any scale or renormalization constant). As will be discussed below, in the Abelian U(1) model with fermions, these requirements can be formulated in diagrammatic language and are described by the blocks of Feynman graphs, where the QED coupling constant $a=\alpha / \pi$ is fixed and is not renormalized. This leads to the property $Z_{3}=1$, where $Z_{3}$ is the renormalization constant of the photon propagator, which is related to the renormalization of the QED coupling constant by $a=Z_{3} a^{B}$, where $a=\alpha / \pi$. In this QED-type model, characterized by the approximation $Z_{3}=1$, the RG $\beta$-function is identically equal to zero, namely

$$
\beta(a)=\left.\mu^{2} \frac{\partial a}{\partial \mu^{2}}\right|_{(a)}{ }^{B} \text { fixed }=\mu^{2} \frac{\partial \ln Z_{3}}{\partial \mu^{2}}=0 .
$$

This property is equivalent to the existence of the CS in this approximation, which as in the case of $\mathcal{N}=4 \mathrm{SYM}$ theory leads to vanishing of the RG $\beta$-function in all orders of perturbation theory.

In the case of an $\mathrm{SU}\left(N_{c}\right)$ gauge group, when the renormalized coupling constant $a_{s}=\alpha_{s} / \pi$ can be defined through the renormalization of several vertexes, namely through triple-gluon vertex, four-gluon vertex or quark-antiquark-gluon vertex, it is unclear how to formulate the CS limit in a manner similar to that discussed above in the case of the U(1)-model, i.e. considering sets of specific Feynman graphs.

Since the basic requirement $a_{s}=a_{s}^{B}$ of this limit does not depend from the scale parameter the $\mathrm{RG} \beta$-function of this $\mathrm{SU}\left(N_{c}\right)$-based model is identically equal to zero in all orders of perturbation theory

$$
\beta\left(a_{s}\right)=\left.\mu^{2} \frac{\partial a_{s}}{\partial \mu^{2}}\right|_{a_{s}^{B} \text { fixed }}=0
$$


The notion of the CS limit of the gauge models turn out to be very useful for deriving the relations between concrete analytical scale-independent perturbative approximations for the coefficient functions of the Bjp, EJ and the GLS sum rules and the similar approximations of the $D$-functions, constructed from the NS vector currents and the SI vector currents. Note. that this consideration presumes, that the renormalization constant of the SI axial-vector current $A_{\mu}(x)=Z^{\mathrm{SI}}\left(a_{s}\right) A_{\mu}^{B}(x)$ is fixed as $Z^{\mathrm{SI}}=1$.

The basic property, which will be used in the derivation of the relations mentioned above, is that in the CS limit the three-point Green functions of eq. (1.2) and eq. (1.3) have 1-loop expressions, which are identical to that of the AVV Green function in eq. (1.1), namely

$$
\begin{aligned}
& \tilde{T}_{\mu \alpha \beta}^{a b}(p, q)=\delta^{a b} \Delta_{\mu \alpha \beta}^{1-l}(p, q) \\
& \tilde{\tilde{T}}_{\mu \alpha \beta}^{a b}(p, q)=N_{F} \delta^{a b} \Delta_{\mu \alpha \beta}^{1-l}(p, q)
\end{aligned}
$$

where the 1-loop contributions $\Delta_{\mu \alpha \beta}^{1-l}(p, q)$ on the r.h.s. of eq. (1.1), eq. (2.3) and eq. (2.4) are the same and $N_{F}$ in eq. (2.4) appears in view of the fact that the $S I$ vector current in eq. (1.3) is defined as $V_{\beta}(x)=\bar{\psi}_{i}(x) \gamma_{\beta} \psi_{i}(x)$ where $i$ counts the number of the fermion flavours with identical charges and thus can be re-written as $V_{\beta}(x)=N_{F} \bar{\psi}(x) \gamma_{\beta} \psi(x)$. As will be demonstrated, in the CS limit the application of the operator product expansion (OPE) approach to eq. (1.1), eq. (2.3) and eq. (2.4) allow us to derive relations between the approximations for the coefficient functions of the DIS sum rules, which will be defined below.

\subsection{The definitions of the perturbative coefficient functions}

Within perturbation theory the Bjorken sum rule of polarized lepton-nucleon DIS is defined by

$$
S_{\mathrm{Bjp}}\left(a_{s}\left(Q^{2}\right)\right)=\int_{0}^{1}\left(g_{1}^{l p}\left(x, Q^{2}\right)-g_{1}^{l n}\left(x, Q^{2}\right)\right) d x=\frac{1}{6} g_{A} C_{\mathrm{Bjp}}\left(a_{s}\left(Q^{2}\right)\right)
$$

where $g_{A}$ is the axial nucleon coupling constant.

In the $\overline{M S}$-scheme the expression for the polarized Ellis-Jaffe sum rule is

$$
\begin{aligned}
E J^{l p(n)}\left(Q^{2}\right)= & \int_{0}^{1} g_{1}^{l p(n)}\left(x, Q^{2}\right) d x \\
= & C_{\mathrm{EJ}}^{\mathrm{NS}}\left(a_{s}\left(Q^{2}\right)\right)\left( \pm \frac{1}{12} a_{3}+\frac{1}{36} a_{8}\right) \\
& \quad+C_{\mathrm{EJ}}^{\mathrm{SI}}\left(a_{s}\left(Q^{2}\right)\right) \exp \left(\int_{a_{s}\left(\mu^{2}\right)}^{a_{s}\left(Q^{2}\right)} \frac{\gamma_{\mathrm{SI}}(x)}{\beta(x)} d x\right) \frac{1}{9} \Delta \Sigma\left(\mu^{2}\right)
\end{aligned}
$$

Here $a_{3}=\Delta u-\Delta d=g_{A}, a_{8}=\Delta u+\Delta d-2 \Delta s, \Delta \Sigma=\Delta u+\Delta d+\Delta s$ and $\Delta u, \Delta d$ and $\Delta s$ are the polarized parton distributions, while the subscripts $l p(n)$ labels the structure functions $g_{1}\left(x, Q^{2}\right)$ of polarized DIS of charged leptons $(l)$ on protons $(p)$ and neutrons $(n)$. Note that the polarized gluon distribution $\Delta G$, introduced in QCD in refs. [42, 43] does 
not contribute to eq. (2.6) in the $\overline{M S}$-scheme [44]. The perturbative expression for the Gross-Llewellyn Smith sum rule of neutrino-nucleon DIS can be defined as

$$
S_{\mathrm{GLS}}\left(a_{s}\right)=\frac{1}{2} \int_{0}^{1} F_{3}^{\nu p+\bar{\nu} p}\left(x, Q^{2}\right) d x=3 C_{\mathrm{GLS}}\left(a_{s}\left(Q^{2}\right)\right) .
$$

Considering the correlator of two NS axial-vector currents

$$
i \int<0\left|T\left(A_{\mu}^{a}(x) A_{\rho}^{b}(0)\right)\right| 0>e^{i q x} d^{4} x=\delta^{a b}\left(q_{\mu} q_{\rho}-g_{\mu \rho} q^{2}\right) \Pi^{\mathrm{NS}}\left(a_{s}\left(\mu^{2}\right), Q^{2} / \mu^{2}\right),
$$

one can define the Adler $D$-function of the NS axial-vector currents as

$$
D_{\mathrm{NS}}\left(a_{s}\left(Q^{2}\right)\right)=-12 \pi^{2} Q^{2} \frac{d \Pi^{\mathrm{NS}}\left(a_{s}\left(\mu^{2}\right), Q^{2} / \mu^{2}\right)}{d Q^{2}}=d_{R} C_{D}^{\mathrm{NS}}\left(a_{s}\left(Q^{2}\right)\right)
$$

where $d_{R}$ is the dimension of the quark representation and $\mu^{2}=Q^{2}$. The correlator of the two SI vector quark currents has the following transverse form

$$
i \int<0\left|T\left(V_{\mu}(x) V_{\rho}(0)\right)\right| 0>e^{i q x} d^{4} x=\left(q_{\mu} q_{\rho}-g_{\mu \rho} q^{2}\right) \Pi^{V}\left(a_{s}\left(\mu^{2}\right), Q^{2} / \mu^{2}\right) \quad .
$$

The corresponding Adler $D$ can be defined as

$$
D_{V}\left(a_{s}\left(Q^{2}\right)\right)=-12 \pi^{2} Q^{2} \frac{d \Pi^{V}\left(a_{s}\left(\mu^{2}\right), Q^{2} / \mu^{2}\right)}{d Q^{2}}=N_{F} C_{D}^{V}\left(a_{s}\left(Q^{2}\right)\right),
$$

where $N_{F}$ is number of fermion species. Note, that in eq. (2.10) the expression for the $S I$ fermion vector current is fixed as $V_{\mu}=\bar{\psi}_{i} \gamma_{\mu} \psi_{i}$, which leads to the appearance of the factor $N_{F}$ in the r.h.s. of eq. (2.11). This particular definition is useful for considering the cases of both $\mathrm{SU}\left(N_{c}\right)$ and $\mathrm{U}(1)$ expressions of eq. (2.11) in the same manner without introducing quark charges $Q_{i}$ and thus changing the SI quark vector current in eq. (2.10) to the electromagnetic quark current $J_{\mu}^{E M}=Q_{i} \bar{\psi}_{i} \gamma_{\mu} \psi_{i}$.

The coefficient function of the RG-invariant quantities defined above obey the following RG equations

$$
\begin{aligned}
& \left(\mu^{2} \frac{\partial}{\partial \mu^{2}}+\beta\left(a_{s}\right) \frac{\partial}{\partial a_{s}}\right) C_{\mathrm{Bjp}}\left(a_{s}\left(\mu^{2}\right), Q^{2} / \mu^{2}\right)=0 \\
& \left(\mu^{2} \frac{\partial}{\partial \mu^{2}}+\beta\left(a_{s}\right) \frac{\partial}{\partial a_{s}}\right) C_{\mathrm{EJ}}^{\mathrm{NS}}\left(a_{s}\left(\mu^{2}\right), Q^{2} / \mu^{2}\right)=0 \\
& \left(\mu^{2} \frac{\partial}{\partial \mu^{2}}+\beta\left(a_{s}\right) \frac{\partial}{\partial a_{s}}\right) C_{\mathrm{GLS}}\left(a_{s}\left(\mu^{2}\right), Q^{2} / \mu^{2}\right)=0 \\
& \left(\mu^{2} \frac{\partial}{\partial \mu^{2}}+\beta\left(a_{s}\right) \frac{\partial}{\partial a_{s}}\right) C_{D}^{\mathrm{NS}}\left(a_{s}\left(\mu^{2}\right), Q^{2} / \mu^{2}\right)=0 \\
& \left(\mu^{2} \frac{\partial}{\partial \mu^{2}}+\beta\left(a_{s}\right) \frac{\partial}{\partial a_{s}}\right) C_{D}^{V}\left(a_{s}\left(\mu^{2}\right), Q^{2} / \mu^{2}\right)=0
\end{aligned}
$$

where $Q^{2}=-q^{2}$ is the Euclidean momentum transfer, $\mu^{2}$ is the scale parameter of the $\overline{M S}$-scheme. The coefficient function of the $D$-function of vector currents and of the GLS sum rule contain the NS and SI-type contributions, namely

$$
\begin{aligned}
C_{D}^{V}\left(a_{s}\left(\mu^{2}, Q^{2} / \mu^{2}\right)\right) & =C_{D}^{\mathrm{NS}}\left(a_{s}\left(\mu^{2}, Q^{2} / \mu^{2}\right)\right)+N_{F} C_{D}^{\mathrm{SI}}\left(a_{s}\left(\mu^{2}, Q^{2} / \mu^{2}\right)\right) ; \\
C_{\mathrm{GLS}}\left(a_{s}\left(\mu^{2}, Q^{2} / \mu^{2}\right)\right) & =C_{\mathrm{GLS}}^{\mathrm{NS}}\left(a_{s}\left(\mu^{2}, Q^{2} / \mu^{2}\right)\right)+N_{F} C_{\mathrm{GLS}}^{\mathrm{SI}}\left(a_{s}\left(\mu^{2}, Q^{2} / \mu^{2}\right)\right) .
\end{aligned}
$$


where in both eq. (2.17) and eq. (2.18) the SI-type perturbative corrections appear at the $a_{s}^{3}$-level (see the works of refs. [27-30] and ref. [26] correspondingly).

As was found recently [37], the coefficient function of the Bjp sum rule also contains SI-type contributions

$$
C_{\mathrm{Bjp}}\left(a_{s}\left(\mu^{2}, Q^{2} / \mu^{2}\right)\right)=C_{\mathrm{Bjp}}^{\mathrm{NS}}\left(a_{s}\left(\mu^{2}, Q^{2} / \mu^{2}\right)+C_{\mathrm{Bjp}}^{\mathrm{SI}}\left(a_{s}\left(\mu^{2}, Q^{2} / \mu^{2}\right)\right)\right.
$$

which appear first at the level of the $a_{s}^{4}$ corrections. It will be demonstrated that the NS contributions to the coefficient functions of the DIS sum rules coincide in all-orders of perturbation theory, namely that

$$
C_{\mathrm{GLS}}^{\mathrm{NS}}\left(a_{s}\left(\mu^{2}\right), Q^{2} / \mu^{2}\right)=C_{\mathrm{Bjp}}^{\mathrm{NS}}\left(a_{s}\left(\mu^{2}\right), Q^{2} / \mu^{2}\right)=C_{\mathrm{EJ}}^{\mathrm{NS}}\left(a_{s}\left(\mu^{2}\right), Q^{2} / \mu^{2}\right) .
$$

These coefficient functions are power series in $a_{s}=\alpha_{s} / \pi$, where $\alpha_{s}$ is the coupling constant of the $\mathrm{SU}\left(N_{c}\right)$ colour gauge theory. In general the variation of $a_{s}$ is governed by RG $\beta$ function of the $\mathrm{SU}\left(N_{c}\right)$ gauge group, which is defined as

$$
\beta\left(a_{s}\right)=\left.\mu^{2} \frac{\partial a_{s}}{\partial \mu^{2}}\right|_{\left(a_{s}\right)^{B}} \text { fixed }=-\sum_{k \geq 0} \beta_{k} a_{s}^{k+2} .
$$

The RG equation for the SI coefficient function for the EJ sum rule contains the anomalous dimension function, namely

$$
\left(\mu^{2} \frac{\partial}{\partial \mu^{2}}+\beta\left(a_{s}\right) \frac{\partial}{\partial a_{s}}+\gamma^{\mathrm{SI}}\left(a_{s}\right)\right) C_{\mathrm{EJ}}^{\mathrm{SI}}\left(a_{s}\left(\mu^{2}\right), Q^{2} / \mu^{2}\right)=0 .
$$

The anomalous dimension of the SI axial current $A_{\mu}$ is defined as

$$
\gamma^{\mathrm{SI}}\left(a_{s}\right)=\mu^{2} \frac{\partial \ln Z^{\mathrm{SI}}\left(a_{s}\right)}{\partial \mu^{2}}=\sum_{l \geq 0} \gamma_{l} a_{s}^{l+1}
$$

where $A_{\mu}=Z^{\mathrm{SI}}\left(a_{s}\right) A_{\mu}^{B}$. This anomalous dimension enters the four-loop calculations of ref. [45], though its analytical expression in the $\overline{M S}$-scheme is known at the three-loop level only [46] and can be re-written as

$$
\begin{aligned}
\gamma^{\mathrm{SI}}\left(a_{s}\right)=-\frac{3}{4} C_{F} T_{F} N_{F} a_{s}^{2}+\left(-\frac{71}{48}\right. & C_{A} C_{F} T_{F} N_{F} \\
& \left.+\frac{1}{12} C_{F}\left(T_{F} N_{F}\right)^{2}+\frac{9}{16} C_{F}^{2}\left(T_{F} N_{F}\right)\right) a_{s}^{3}+O\left(a_{s}^{4}\right) .
\end{aligned}
$$

In eq. (2.24) $\gamma_{0}$ is zero due to the fulfilment of the Ward identities for the SI axial vector current, $C_{F}$ and $C_{A}$ are the Casimir operators, $N_{F}$ counts the number of flavours, $T_{F}=1 / 2$ is the normalization factor, or the Dynkin index, which will be defined below.

\subsection{The definitions of the $\mathrm{SU}\left(N_{c}\right)$ and $\mathrm{U}(1)$ group weights}

In order to recall how one can transform perturbative series for the case of the non-Abelian $\mathrm{SU}\left(N_{c}\right)$ gauge model to the case of the Abelian $\mathrm{U}(1)$ theory, we follow in this section the 
studies, performed in refs. [47-50], and present the general definitions of the Casimir operators and the structure constants for the $\mathrm{SU}\left(N_{c}\right)$ and $\mathrm{U}(1)$ gauge groups. The generators $T^{a}$ of the Lie algebra of the $\mathrm{SU}\left(N_{c}\right)$ group satisfy the following commutation relations

$$
\left[T^{a}, T^{b}\right]=i f^{a b c} T^{c}
$$

where $f^{a b c}$ is the antisymmetric structure constant. In a fermion representation, which contains $N_{F}$ fermions, one has

$$
T^{a} T^{a}=C_{F} I .
$$

Here $I$ is the unit matrix and $C_{F}$ is the quadratic Casimir operator of the Lie algebra. The Casimir operator $C_{A}$ of its adjoint representation is defined as

$$
f^{a c d} f^{b c d}=C_{A} \delta^{a b}
$$

The Dynkin index $T_{F}$ of the Lie algebra of the $\mathrm{SU}\left(N_{c}\right)$ group has the following form

$$
\operatorname{Tr}\left[T^{a} T^{b}\right]=T_{F} \delta^{a b}
$$

Using eq. (2.26) and eq. (2.27) one gets

$$
C_{F} d_{F}=T_{F} N_{A}
$$

where $N_{A}$ is the number of the generators $T^{a}$, and $d_{F}$ is the dimension of the fundamental representation $R$ of the $\mathrm{SU}\left(N_{c}\right)$ gauge group Lie algebra. The totally symmetric tensor $d_{F}^{a b c}$, which already appeared in the discussions presented above, is defined as

$$
d_{F}^{a b c}=\frac{1}{2} \operatorname{Tr}\left[T^{a} T^{b} T^{c}+T^{a} T^{c} T^{b}\right]
$$

The generators $T^{a}$ of the $\mathrm{SU}\left(N_{c}\right)$ colour gauge group are related to the colour matrixes $\lambda^{a}$ as $T^{a}=\lambda^{a} / 2$. In the fundamental representation $R$ the concrete analytical expressions of the introduced above $\mathrm{SU}\left(N_{c}\right)$ group characteristics are

$$
C_{F}=\frac{N_{c}^{2}-1}{2 N_{c}}, C_{A}=N_{c} \quad ; \quad N_{A}=N_{c}^{2}-1 \quad ; \quad T_{F}=\frac{1}{2} \quad, \quad d_{F}=N_{c} \quad .
$$

In this representation the product of two totally symmetric tensors $d_{F}^{a b c}$ equals to

$$
d_{F}^{a b c} d_{F}^{a b c}=\left(\frac{N_{c}^{2}-4}{N_{c}}\right)\left(N_{c}^{2}-1\right) .
$$

It was was first obtained by the authors of ref. [27] using the original method of ref. [47]. This expression was confirmed later on in the detailed work of ref. [49].

In the case of the Abelian U(1) gauge group with fermions one has

$$
f^{a b c}=0 \quad, \quad T^{a}=1, \quad N_{A}=1 .
$$

Thus, using (2.26) and (2.27) we get that in the $\mathrm{U}(1)$ model $C_{F}=1$ and $C_{A}=0$. Rewriting (2.29) as

$$
T_{F}=\left(C_{F} d_{F}\right) / N_{A}
$$




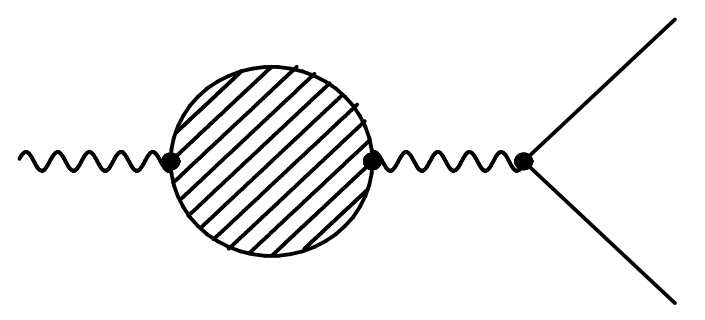

Figure 1. The set of photon vacuum polarization graphs which is renormalizing the charge in the pqQED model as specified below.

taking into account that in QED $d_{F}=1, N_{A}=1$ one obtains that for the Lie algebra of the $\mathrm{U}(1)$ group the Dynkin index is fixed as

$$
T_{F}=1
$$

The absence of colour structure in the QED vector current $J_{\mu}(x)=\bar{\psi}(x) \gamma_{\mu} \psi(x)$ and the application of eq. (2.30) implies that in QED $d_{F}^{a b c}=1$.

To summarize

$$
C_{F}=1, \quad C_{A}=0 \quad ; \quad T_{F}=1, \quad f^{a b c}=0 \quad, \quad d_{F}^{a b c}=1 \quad, \quad d_{F}=1
$$

are the QED ( or of the Abelian U(1) group) analogs of the group structures, which appear in the multiloop calculations in the non-Abelian $\mathrm{SU}\left(N_{c}\right)$ group.

\subsection{Specification of the conformally invariant limit in perturbative QED}

To clarify the notion of the CS limit of the gauge models with fermions to be used in this work, which does not correspond to the fixed points or "conformally invariant windows" in the expressions for the RG $\beta$-functions of these models, we consider first the case of QED, and the perturbative quenched QED (pqQED) model in particular. As was recalled above, it was used some time ago in studies of the finite QED program. This model is described by the set of QED graphs without internal vacuum polarization insertions in various multiloop diagrams. Among these diagrams is the photon-electron-positron vertex, which defines the renormalization of charge in QED. In the pqQED model the external photon line of this photon-electron-positron vertex, depicted in figure 1, is renormalized by the multiloop photon vacuum polarization function with the single external fermion loop only.

Of course, there are also the diagram with the insertion of the pqQED approximation of the photon propagators into external lepton lines of the photon-lepton-lepton vertex and into the vertex itself. They are also contributing into the pqQED approximation of the renormalization constants $Z_{2}$ and $Z_{1}$. But due to the Ward identities $Z_{1}$ and $Z_{2}$ are cancelling each other, so only the diagrams of figure 1 are surviving in the definition of the renormalized coupling constant of QED and pqQED approximation.

Thus the pqQED approximation of the photon vacuum polarization function is defined by the one-particle irreducible vacuum polarization function with the exclusion of diagrams with lepton-loop inserions into internal photon line (see figure 2). 


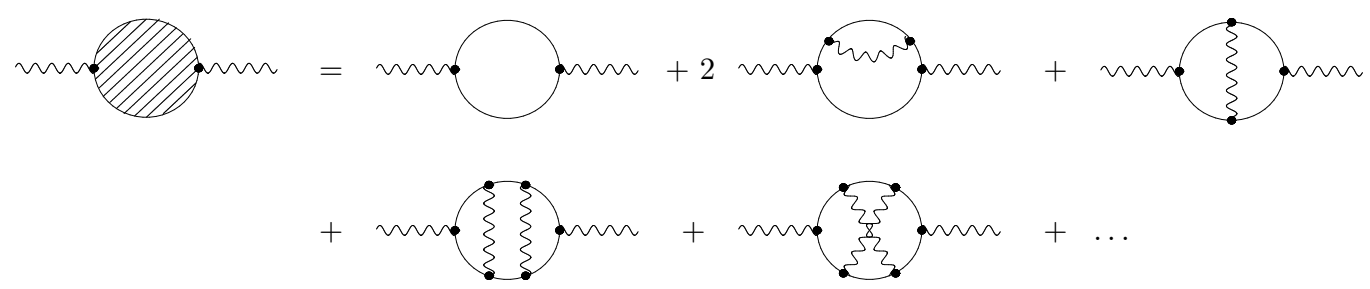

Figure 2. The pqQED approximation of the photon vacuum polarization function which do not contain the graphs with lepton-loop insertions into internal photon lines.

In the approximation considered the renormalization constant $Z_{3}$ of the photon propagator and the related RG $\beta$-function are defined by the photon vacuum polarization graphs with one external fermion loop.

Within pqQED model with $N_{F}=N$ number of leptons the expression for the $\beta$ function can be written down as

$$
\beta_{\mathrm{pqQED}}(a)=\mu^{2} \frac{\partial a}{\partial \mu^{2}}=\sum_{k \geq 0} \beta_{k}^{[1]} N a^{k+2}
$$

where $a=\alpha / \pi, \alpha$ is the renormalized coupling constant of the pqQED model, and the coefficients $\beta_{k}^{[1]}$ do not depend from the number of leptons $N$. At the 4-loop level these results follow the analytical calculations of ref. [19], which were directly confirmed within the pqQED model by the calculations of ref. [51].

The analytical result for the 5-loop coefficient $\beta_{4}^{[1]}$ was announced in ref. [52] and published later on in ref. [31] after performing the calculational cross-check outlined in ref. [53]. Note, that since there are no sub-divergencies in the total pqQED expression for the photon vacuum polarization function, the $\beta$-function of pqQED, as defined in eq. (2.37), does not depend on the choice of the subtractions scheme to all orders of perturbation theory. Therefore, pqQED approximation is an example of a model, where the remaining scale dependence manifests itself in the perturbative expressions for the related massless Green functions only.

Let us now move one step further and define the conformal invlimit of perturbative QED. It is realized when there is no scale in the theory, which is introduced by charge renormalization. Within the language of renormalization constants this happens when $Z_{3}=1$. This approximation is equivalent to the case when $a=a^{B}$ and $\beta(a)=\beta\left(a^{B}\right)=0$.

The conditions fixed above define the CS limit of QED considered in this work in diagrammatic language. It differs from the CS limit which is restored in ref. [41] by the modification of the QED Lagrangian in an arbitrary number of dimensions.

To summarize in our case the conformally invariant limit of perturbative QED exists

1. in the approximation when only the Feynman diagrams contributing to Green functions without fermion loop insertions into internal photon lines are considered; 
2. this approximation should be combined with the theoretical requirement that in the concrete perturbative expansions one should use not the running coupling, but the bare parameter $a=\alpha / \pi$;

3. the latter property holds when in the photon-lepton-anti-lepton vertex diagrams the photon vacuum polarization insertions (even with single fermion loop) are not considered.

In other words, in the conformally invariant limit of QED considered the photon vacuum polarization function of pqQED involves the two-point Green function of vector vector currents, while in the vertex Green functions for the dressed photon-lepton- anti-lepton vertex they are neglected.

In the talk [22] the conformally invariant limit of QED specified above was used to outline the derivation of an all-order identity between special contributions to the NS and SI coefficient functions for the Ellis-Jaffe sum rule of polarized lepton-nucleon DIS. The NS and SI coefficient functions $C_{\mathrm{EJ}}^{\mathrm{NS}}\left(a_{s}\right)$ and $C_{\mathrm{EJ}}^{\mathrm{SI}}\left(a_{s}\right)$ enter the operator product expansion expansion of the $T$-product of two NS vector currents as

$$
\begin{aligned}
\left.i \int T\left(V_{\alpha}^{a}(x) V_{\beta}^{b}(0)\right) e^{i p x} d^{4} x\right|_{p^{2} \rightarrow \infty}= & \delta^{a b}\left(p_{\alpha} p_{\beta}-g_{\alpha \beta} p^{2}\right) \Pi^{\mathrm{NS}}\left(a_{s}\left(\mu^{2}\right), P^{2} / \mu^{2}\right) \\
& +d^{a b d} \epsilon_{\alpha \beta \rho \sigma} \frac{p^{\sigma}}{P^{2}} C_{\mathrm{EJ}}^{\mathrm{NS}}\left(a_{s}\left(\mu^{2}\right), P^{2} / \mu^{2}\right) A_{\rho}^{d}(0) \\
& +\delta^{a b} \epsilon_{\alpha \beta \rho \sigma} \frac{p^{\sigma}}{P^{2}} C_{\mathrm{EJ}}^{\mathrm{SI}}\left(a_{s}\left(\mu^{2}\right), P^{2} / \mu^{2}\right) A_{\rho}(0) \\
& + \text { higher twist terms }
\end{aligned}
$$

where $P^{2}=-p^{2}$ is the Euclidean transfer momentum and $\mu^{2}$ is the renormalization scale parameter. The second term of the r.h.s. of eq. (2.38) also defines the NS part in the coefficient function $C_{\mathrm{Bjp}}^{\mathrm{NS}}\left(a_{s}\right)$ of the Bjp sum rule [54], which was introduced above in eq. (2.5). Therefore, one has $C_{\mathrm{EJ}}^{\mathrm{NS}}\left(a_{s}\right)=C_{\mathrm{Bjp}}^{\mathrm{NS}}\left(a_{s}\right)$. The analytical expression for the $a_{s^{-}}^{2}$ and $a_{s}^{3}$-corrections to $C_{\mathrm{Bjp}}\left(a_{s}\right)$ were analytically evaluated in the $\overline{M S}$-scheme in the works of ref. [55] and ref. [26] respectively, while the corresponding NS-type $a_{s}^{4}$ contributions were evaluated in ref. [31].

To get the non-zero pqQED analog of the $O\left(a_{s}^{3}\right)$ approximation of the anomalous dimension $\gamma^{\mathrm{SI}}\left(a_{s}\right)$, defined in eq. (2.24), we use the discussions of section 2.4, fixing $C_{F}=1$, $C_{A}=0, T_{F}=1$ (as follows from eq. (2.36)), and take $N_{F}^{k}=0$ for $k \geq 2$ into its available $\mathrm{SU}\left(N_{c}\right)$ expression of eq. (2.24). In order to put it to zero as a whole and thus move to the case of CS approximation of perturbative QED, it is necessary to add to the requirements (1)-(3), introduced above while specifying the conformal invariant limit of perturbative QED, the additional condition of non-renormalization of the SI axial-vector current, i.e. the condition $A_{\mu}(x)=A_{\mu}^{B}(x)$.

In this case the correlator of two SI bare axial-vector currents contains a single external lepton loop and has the transverse form, namely

$$
i \int<0\left|T\left(A_{\mu}^{B}(x)\right)\left(A_{\rho}^{B}(0)\right)\right| 0>e^{i q x} d^{4} x=\left(g_{\mu \rho} q^{2}-q_{\mu} q_{\rho}\right) \Pi^{\mathrm{SI}}\left(a^{B}, Q^{2} / \mu^{2}\right) .
$$


The corresponding multiloop approximation of the formfactor $\Pi^{\mathrm{SI}}\left(a^{B}, Q^{2} / \mu^{2}\right)$ does not contain the diagrams with triangle contributions to the external bare vertex, and therefore the anomalous dimension $\gamma_{\mathrm{SI}}(a)$, discussed in the related QED studies of ref. [14], is absent. Moreover, in the conformal invariant limit of perturbative QED the formfactor $\Pi^{\mathrm{SI}}\left(a^{B}, Q^{2} / \mu^{2}\right)$ coincides with the conformal-invariant approximation for the formfactor $\Pi^{\mathrm{NS}}\left(a^{B}, Q^{2} / \mu^{2}\right)$ of two NS axial -vector currents, which appears in the QED version of eq. (2.39).

In the case of perturbative QCD or, more generally, in the $\mathrm{SU}\left(N_{c}\right)$ gauge model with fermions, the initial CS of the massless quark-parton model can be restored by fixing $a_{s}=a_{s}^{B}$ and $A_{\mu}(x)=A_{\mu}^{B}(x)$. Using the definitions of eq. (2.21) and eq. (2.24) one gets identically zero expressions for the RG functions $\beta\left(a_{s}\right)=0$ and $\gamma^{\mathrm{SI}}\left(a_{s}\right)=0$ and the expression of the transverse expression of the two-point Green function of the bare axial-vector currents $A_{\mu}^{B}(x)$, which has the form, similar to the one of (2.39).

These pure theoretical conditions are useful for the derivations of all-order perturbative identities between the concrete terms in the NS and SI coefficient functions of the EJ sum rule and the NS coefficient function of the Bjp sum rule and the GLS sum rule of the $\nu N$ DIS. In the $\overline{M S}$-scheme the total coefficient function of the GLS sum rule $C_{\mathrm{GLS}}\left(a_{s}\right)$ is defined through the OPE of the NS axial-vector and vector fermion currents (see e.g. [54]) as

$$
i \int T A_{\mu}^{a}(x) V_{\nu}^{b}(0) e^{i p x} d x=\delta^{a b} \epsilon_{\mu \nu \alpha \beta} \frac{p^{\beta}}{P^{2}} C_{\mathrm{GLS}}\left(a_{s}\left(\mu^{2}, P^{2} / \mu^{2}\right)\right) V_{\alpha}(0)+\text { higher twist terms. }
$$

where we follow the notation consistent with the notation for the Green function of eq. (1.3). In the next section we will consider theoretical results for the DIS sum rules, which follow from the defined in this work CS limit of the $\mathrm{SU}\left(N_{c}\right)$ and $\mathrm{U}(1)$ gauge models.

\section{Conformal symmetry governed contributions to the coefficient func- tions of the DIS sum rules in QED and QCD}

\subsection{The all order perturbative identities between coefficient functions of DIS sum rules in the conformal symmetry limit}

Let us compare application of the OPE approach to the three AVV three-point Green functions of eq. (1.1), eq. (1.2) and eq. (1.3). In was already discussed in section 1 that in the CS limit all these three AVV three-point Green functions are not renormalized and are expressed through the same one-loop three-point function $\Delta_{\mu \alpha \beta}^{1-l}(p, q)$. The results, obtained in ref. [2] in the kinematic regime $(p q)=0$ (see ref. [56] as well) demonstrate that this three-point function can be expressed through three form-factors:

$$
\begin{aligned}
\Delta_{\mu \alpha \beta}^{1-l}(p, q)= & \xi_{1}^{1-l}\left(p^{2}, q^{2}\right) \epsilon_{\mu \alpha \beta \tau} p^{\tau} \\
& +\xi_{2}^{1-l}\left(p^{2}, q^{2}\right)\left(q_{\alpha} \epsilon_{\mu \beta \rho \tau} p^{\rho} q^{\tau}-q_{\beta} \epsilon_{\mu \alpha \rho \tau} p^{\rho} q^{\tau}\right) \\
& +\xi_{3}^{1-l}\left(p^{2}, q^{2}\right)\left(p_{\alpha} \epsilon_{\mu \beta \rho \tau} p^{\rho} q^{\tau}+p_{\beta} \epsilon_{\mu \alpha \rho \tau} p^{\rho} q^{\tau}\right) .
\end{aligned}
$$

Using this property and applying the OPE expansion to eq. (1.1) and eq. (1.2) in the limit of large $P^{2}$ and taking into account the definition of eq. (2.39), we get the following 
two expressions

$$
\begin{aligned}
& d_{R}^{a b c} \Delta_{\mu \alpha \beta}^{1-l}(p, q)=i d_{R}^{a b d} \epsilon_{\alpha \beta \rho \delta} \frac{p^{\delta}}{P^{2}} C_{\mathrm{EJ}}^{\mathrm{NS}}\left(a_{s}^{B}\right) \int<0\left|T\left(A_{\mu}^{c}(x) A_{\rho}^{d}(0)\right)\right| 0>e^{i q x} d^{4} x \\
& \delta^{a b} \Delta_{\mu \alpha \beta}^{1-l}(p, q)=i \delta^{a b} \epsilon_{\alpha \beta \rho \delta} \frac{p^{\delta}}{P^{2}} C_{\mathrm{EJ}}^{\mathrm{SI}}\left(a_{s}^{B}\right) \int<0\left|T\left(A_{\mu}^{B}(x) A_{\rho}^{B}(0)\right)\right| 0>e^{i q x} d^{4} x
\end{aligned}
$$

where the correlators of the NS and SI axial-vector currents do not contain diagrams with insertions responsible for renormalization of the internal propagators of gauge particles, and the $\mathrm{SU}\left(N_{c}\right)$-group coupling constant $a_{s}=a_{s}^{B}$.

Combining now eq. (3.1), eq. (3.2) and eq. (3.3) and taking into account that in the CS limit the l.h.s. of eqs. (3.2), (3.3) do not contain radiative corrections and that in the $\mathrm{SU}\left(N_{c}\right)$ gauge model the correlators of the NS axial-vector currents $A_{\mu}^{a}(x)$ and of the bare SI axial-vector currents $A_{\mu}^{B}$ are transverse (see eq. (2.8) and the $\mathrm{SU}\left(N_{c}\right)$-group analog of eq. (2.39)) we get

$$
\left.\xi_{2}^{1-l o o p}\left(q^{2}, p^{2}\right)\right|_{\left|p^{2}\right| \geq\left|q^{2}\right| \gg 0}=\frac{1}{p^{2}} C_{\mathrm{EJ}}^{\mathrm{SI}}\left(a_{s}^{B}\right) \Pi^{\mathrm{SI}}\left(a_{s}^{B}, Q^{2} / \mu^{2}\right)
$$

This expression is similar to the CS limit of the expression obtained in ref. [2] in the NS axial-vector channel, i.e.

$$
\left.\xi_{2}^{1-l o o p}\left(q^{2}, p^{2}\right)\right|_{\left|p^{2}\right| \geq\left|q^{2}\right| \gg 0}=\frac{1}{p^{2}} C_{\mathrm{EJ}}^{\mathrm{NS}}\left(a_{s}^{B}\right) \Pi^{\mathrm{NS}}\left(a_{s}^{B}, Q^{2} / \mu^{2}\right)
$$

Note, that the remaining $Q^{2} / \mu^{2}$-dependence comes from the single $\log \left(Q^{2} / \mu^{2}\right)$-terms, which are related to the overall divergence of the single-fermion loop approximations of the correlators of NS and SI axial-vector currents. Taking now the "weighted" derivatives $-Q^{2}\left(d / d Q^{2}\right)$ in (3.4) and (3.5) we get the following pair of the Crewther-type identities

$$
\begin{gathered}
C_{\mathrm{EJ}}^{\mathrm{SI}}\left(a_{s}\right) \times C_{D}^{\mathrm{SI}}\left(a_{s}\right)=\mathbf{1} \\
C_{\mathrm{EJ}}^{\mathrm{NS}}\left(a_{s}\right) \times C_{D}^{\mathrm{NS}}\left(a_{s}\right)=\mathbf{1},
\end{gathered}
$$

where $a_{s}=a_{s}^{B}$ is considered as the fixed parameter. Taking into account the existence of the property

$$
C_{D}^{\mathrm{SI}}\left(a_{s}^{B}\right) \equiv C_{D}^{\mathrm{NS}}\left(a_{s}^{B}\right)
$$

which is fulfilled in all orders of perturbation theory, and that $C_{\mathrm{EJ}}^{\mathrm{NS}}\left(a_{s}\right)=C_{\mathrm{Bjp}}^{\mathrm{NS}}\left(a_{s}\right)$ (for discussions see section 2.2 ), we get the following CS based all-order relation for the coefficient functions of DIS sum rules

$$
C_{\mathrm{DIS}}^{\mathrm{CS}}\left(a_{s}^{B}\right) \equiv C_{\mathrm{EJ}}^{\mathrm{SI}}\left(a_{s}^{B}\right) \equiv C_{\mathrm{EJ}}^{\mathrm{NS}}\left(a_{s}^{B}\right) \equiv C_{B j}^{\mathrm{NS}}\left(a_{s}^{B}\right) \equiv C_{\mathrm{GLS}}^{\mathrm{NS}}\left(a_{s}^{B}\right)
$$

Note, that in the CS limit the ratios of the corresponding approximations for the EJ and Bjp sum rules, which are determined using their definitions from section 2.2 and the CS identities of eq. (3.9), give us the the following relation

$$
\frac{E J^{l p(n)}\left(Q^{2}\right)}{\operatorname{Bjp}\left(Q^{2}\right)}= \pm \frac{1}{2}+\frac{a_{8}}{6 a_{3}}+\frac{2 \Delta \Sigma}{3 a_{3}}
$$




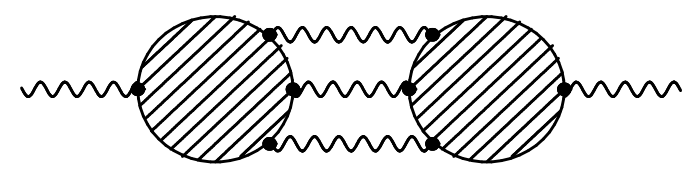

Figure 3. The set of light-by-ligt-type contributions to the photon vacuum polarization function without internal lepton loop insertions.

where $a_{8}=3 a_{3}-4 D, a_{3}, a_{8}$ and $\Delta \Sigma$ were defined above through the polarized parton distributions and $D$ is the hyperon decay constant. These relations coincide with the ones obtained in the massless quark-parton model and can be rewritten as

$$
\frac{E J^{l p}\left(Q^{2}\right)}{B j p\left(Q^{2}\right)}=1+\frac{2(\Delta \Sigma-D)}{3 a_{3}} \quad ; \quad \frac{E J^{l n}\left(Q^{2}\right)}{B j p\left(Q^{2}\right)}=+\frac{2}{3} \frac{(\Delta \Sigma-D)}{a_{3}} .
$$

They lead to the standard quark-parton model definition of the Bjp sum rule through the EJ sum rules, namely

$$
B j p \equiv E J^{l p}-E J^{l n} .
$$

This gives us confidence in the self-consistency of the considerations presented above.

To get the CS limit expression for the coefficient function of the GLS sum rule, we apply the OPE to eq. (1.3) at large $Q^{2}$. As a result, using the definition of eq. (2.40) in the CS limit of the gauge model under considerations we find that

$$
N_{F} \delta^{a b} \Delta_{\mu \alpha \beta}^{1-l}(p, q)=i \delta^{a b} \epsilon_{\mu \alpha \nu \beta} \frac{q^{\beta}}{Q^{2}} C_{\mathrm{GLS}}\left(a_{s}\left(\mu^{2}\right), Q^{2} / \mu^{2}\right) \int<0\left|T\left(V_{\beta}(x) V_{\nu}(0)\right)\right| 0>e^{-i p x} d^{4} x
$$

The analog of eq. (3.4) now reads

$$
\left.\xi_{2}^{1-l o o p}\left(q^{2}, p^{2}\right)\right|_{\left|q^{2}\right| \geq\left|p^{2}\right| \gg 0}=\frac{1}{q^{2}} C_{\mathrm{GLS}}\left(a_{s}^{B}\right) \Pi^{V}\left(a_{s}^{B}, P^{2} / \mu^{2}\right) .
$$

where $N_{F}$ on the l.h.s. of eq. (3.13) comes from the definition of the three-point function of eq. (1.3). Taking the weighted derivative $-P^{2}\left(d / d P^{2}\right)$ on both sides of eq. (3.14) using the definition of the Adler function of $S I$ vector currents from eq. (2.17) we will also use the discovery of finite QED program studies, that the CS limit is also valid in the case of including the SI-type contributions to the correlator of SI vector fermion currents which contain two light-by-light scattering subgraphs without internal lepton loop insertions (see figure 3).

The dashed light-by-light scattering fermion-loop subgraphs of the diagrams of figure 3 do not contain diagrams with lepton loops insertions into internal photons lines (see figure 4). In the approximation we are interested in these diagrams are subtracted from the one-particle irreducible expressions for the subset of QED diagrams with four external photon lines, coupled to the single lepton loop.

Taking into account these light-by-light scattering graphs, we arrive at the following analog of the Crewther-type relation between the coefficient functions of the GLS sum rule and the Adler function of SI vector currents

$$
\left.\left[\left(C_{\mathrm{GLS}}^{\mathrm{NS}}\left(a_{s}^{B}\right)+N_{F} C_{\mathrm{GLS}}^{\mathrm{SI}}\left(a_{s}^{B}\right)\right) \times\left(C_{D}^{\mathrm{NS}}\left(a_{s}^{B}\right)+N_{F} C_{D}^{\mathrm{SI}}\left(a_{s}^{B}\right)\right)\right]\right|_{\mathrm{CS} \text { limit }}=1 .
$$




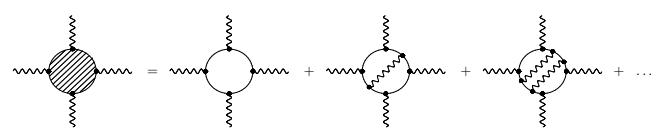

Figure 4. The set of light-by-light scattering graphs without diagrams with internal lepton loop insertions.

It is valid at the level of taking into account $N_{F}$-terms for sure, while the cross-check of the $N_{F}^{2}$ relation requires the evaluation of higher order SI contributions to both $D$-function and to the GLS sum rules, which respects CS limit. At present these require calculations going beyond the level of the analytically evaluated $O\left(a_{s}^{4}\right)$ corrections. Thus, expanding (3.15) in powers of $N_{F}$ we obtain

$$
\begin{array}{r}
C_{\mathrm{GLS}}^{\mathrm{NS}}\left(a_{s}^{B}\right) \times C_{D}^{\mathrm{NS}}\left(a_{s}^{B}\right)=1 \\
C_{\mathrm{GLS}}^{\mathrm{NS}}\left(a_{s}^{B}\right) \times C_{D}^{\mathrm{SI}}\left(a_{s}^{B}\right)+C_{\mathrm{GLS}}^{\mathrm{SI}}\left(a_{s}^{B}\right) \times C_{D}^{\mathrm{NS}}\left(a_{s}^{B}\right)=0
\end{array}
$$

The expression for eq. (3.16) implies that

$$
C_{\mathrm{GLS}}^{\mathrm{NS}}\left(a_{s}\right)=1 / C_{D}^{\mathrm{NS}}\left(a_{s}\right)
$$

and therefore

$$
C_{\mathrm{GLS}}^{\mathrm{NS}}\left(a_{s}^{B}\right)=C_{\mathrm{DIS}}^{\mathrm{CS}}\left(a_{s}^{B}\right)
$$

where the r.h.s. of eq. (3.19) is defined by eq. (3.9). It is identically equal to the conformally invariant contributions to the SI and NS parts of the EJ sum rule and of the NS contributions to the Bjp sum rule. The expression (3.15) is supporting the relation between the $a_{s}^{3} N_{F}$ and the $a_{s}^{4} N_{F} C_{F}$ corrections to the SI contribution into the coefficient function of the GLS sum rule and into the SI contribution to the coefficient function of the $D$-function of $S I$ vector currents. At the $a_{s}^{3}$ - and $a_{s}^{4}$-levels these relations were obtained in ref. [25] and ref. [31] respectively from the results of analytical $a_{s}^{3}$ and $a_{s}^{4}$ calculations.

\subsection{Concrete analytical and numerical results}

Let us present now some concrete expressions for the scale-independent approximations of the several coefficient functions. The first one is the expression for the coefficient function of NS $D$-function, obtained in the conformally-invariant approximation of QED. It follows from the results of direct analytical 5-loop calculations, presented first in the work of ref. [52], discussed in detail in the work of ref. [53] and published later on in ref. [31]. This result has the following form

$$
\begin{aligned}
C_{D}^{\mathrm{NS}} & =1+\frac{3}{4} a-\frac{3}{32} a^{2}-\frac{69}{128} a^{3}+\left(\frac{4157}{2048}+\frac{3}{8} \zeta_{3}\right) a^{4}+O\left(a^{5}\right) \\
& =1+0.75 a-0.094 a^{2}+0.531 a^{3}+2.481 a^{4}+O\left(a^{5}\right) .
\end{aligned}
$$

In the case of the $\mathrm{SU}\left(N_{c}\right)$ model we will consider in this work a similar analytical $O\left(a_{s}^{2}\right)$ expression, which is known from the analysis of ref. [57] (see the work of ref. [58] as well). 
It reads

$$
\begin{aligned}
C_{D}^{\mathrm{NS}}\left(a_{s}\right) & =1+\frac{3}{4} C_{F} a_{s}+\left(-\frac{3}{32} C_{F}^{2}+\frac{1}{16} C_{F} C_{A}\right) a_{s}^{2}+0\left(a_{s}^{3}\right) \\
& =1+a_{s}+\frac{1}{12} a_{s}^{2}+O\left(a_{s}^{3}\right) \\
& =1+a_{s}+0.083 a_{s}^{2}+O\left(a_{s}^{3}\right)
\end{aligned}
$$

where the numerical expressions for the coefficients are obtained in the case of $\mathrm{SU}(3)$ gauge model, namely for $C_{F}=4 / 3$ and $C_{A}=3$. Fixing $C_{F}=1$ and $C_{A}=0$ in ccordance with the discussions, presented in the section 3.2, one can reproduce the related QED results of eq. (3.20) from the ones of eq. (3.22). In the case of QCD the analytical and numerical expressions for the $O\left(a_{s}^{2}\right)$-corrections in eq. (3.23) agree with the results of application of the BLM scale-fixing approach of ref. [59].

In the conformally-invariant limit of perturbative QED the results for the coefficient functions of DIS sum rules can be obtained from the Crewther relations of eq. (3.6) and of eq. (2.7). In this limit the explicit expression for the NS coefficient function of the Bjorken sum rule was first obtained in ref. [53]. Taking it into account we get the following analytical and numerical expressions of the considered in this work identities:

$$
\begin{aligned}
C_{\mathrm{Bjp}}^{\mathrm{NS}}(a) & =C_{\mathrm{EJ}}^{\mathrm{NS}}(a)=C_{\mathrm{EJ}}^{\mathrm{SI}}(a)=C_{\mathrm{GLS}}^{\mathrm{NS}}(a)=1 / C_{D}^{\mathrm{NS}}(a) \\
& =1-\frac{3}{4} a+\frac{21}{32} a^{2}-\frac{3}{128} a^{3}-\left(\frac{4823}{2048}+\frac{3}{8} \zeta_{3}\right) a^{4}+O\left(a^{5}\right) \\
& =1-0.75 a+0.656 a^{2}-0.0234 a^{3}+2.806 a^{4}+O\left(a^{5}\right)
\end{aligned}
$$

The validity of the identity of eq. (3.25) for the coefficient function $C_{\mathrm{EJ}}^{\mathrm{SI}}(a)$ was explicitely demonstrated in ref. [20] at the level of $a^{3}$ corrections. The results of ref. [20] were obtained combining the analytical 3-loop expressions, which follow from the results of calculations of refs. [26, 45] in the CS limit of QED.

It will be of interest to check the validity of this identity in possible direct analytical 4-loop calculations of $C_{\mathrm{EJ}}^{\mathrm{SI}}(a)$.

We present here also the $O\left(a_{s}^{2}\right)$ CS approximations for the DIS sum rules in the the cases of $\mathrm{SU}\left(N_{c}\right)$ and $\mathrm{SU}(3)$ models, which result from the considerations of ref. [58]:

$$
\begin{aligned}
C_{\mathrm{Bjp}}^{\mathrm{NS}}\left(a_{s}\right) & =C_{\mathrm{EJ}}^{\mathrm{SI}}\left(a_{s}\right)=C_{\mathrm{GLS}}^{\mathrm{NS}}\left(a_{s}\right)=1 / C_{D}^{\mathrm{NS}}\left(a_{s}\right) \\
& =1-\frac{3}{4} C_{F} a_{s}+\left(\frac{23}{32} C_{F}^{2}-\frac{1}{16} C_{F} C_{A}\right) a_{s}^{2}+O\left(a_{s}^{3}\right) \\
& =1-a_{s}+\frac{11}{12} a_{s}^{2}+O\left(a_{s}^{3}\right) \\
& =1-a_{s}+0.917 a_{s}^{2}+O\left(a_{s}^{3}\right)
\end{aligned}
$$

The numerical expression for the $O\left(a_{s}^{2}\right)$-coefficient coincides with the result obtained in ref. [60] using the one-scale $O\left(a_{s}^{3}\right)$ extension of the BLM approach of ref. [59], developed 
in ref. [61]. The detailed studies of the differences of these results with other extensions of the BLM approach, which also have the aim to obtain the expressions for the coefficient functions as a series with scale-independent coefficients [62-64] are on the agenda. The method of ref. [57], developed for the formulation of an all-orders extension of the BLM approach, and the new $\overline{M S}$-scheme generalization of the Crewther relation [58], which presumes the application of the two-fold expansion in terms of powers of the $\beta$-function and the coupling constant itself, are quite useful for the investigation of this problem.

\section{Conclusions}

In this work using the language of the bare unrenormalized parameters of QED and QCD and the properties of the absence of radiative corrections to three AVV three-point Green functions in the case when the conformal symmetry of $\mathrm{U}(1)$ and $\mathrm{SU}\left(N_{c}\right)$ gauge models remain valid, we derived three Crewther-type relations and the identities between coefficient functions of DIS sum rules. We demonstrated how to formulate the conformal-invariant limit of perturbative QED on the diagrammatic language and clarified that it is possible to fix the similar CS limit of the $\mathrm{SU}\left(N_{c}\right)$ model using the language of bare unrenormalized parameters. The concrete checks of the possible analytical evaluations of the fourth-order corrections to the SI coefficient functions in the expressions for the EJ sum rule within the conformal-invariant expansion in the U(1) model with fermions were outlined.

The necessity of comparing the results for the coefficient functions of the DIS sum rule and the $D$-functions, obtained within the conformal-invariant limit of the $\mathrm{SU}\left(N_{c}\right)$ model with fermions, with the the generalizations of the BLM approach were emphasized. These generalizations absorb into the scale of the $\overline{M S}$-scheme coupling constant all factors proportional to the coefficients of the $\beta$-function. These coefficients are responsible for violation of the CS. The CS breaking perturbative effects also manifest themselves in the $\overline{M S}$-scheme generalizations of Crewther relations, discovered, studied, theoretically considered, proved and reformulated in the works of ref. [25], refs. [31, 32], ref. [2], refs. [35, 36] and ref. [58] respectively. The manifestation of these effects through the appearance of loop corrections to the AVV three-point functions starting from the 3-loop level are supported by the manifestation of a concrete $\beta_{0} a_{s}^{3}$ dependent correction in the explicitly evaluated 3-loop expressions for the transverse form-factors of the AVV correlator [65]. More detailed studies of various expressions for the generalized Crewther relations should be quite useful for the task of comparing with existing generalizations of the BLM approach.

Another aim of this work was to demonstrate that the principles of the CS, which at present are widely considered in the applications of the OPE to the Green functions in $\mathcal{N}=4$ SUSY Yang Mills models, may have an analogy with the investigations of the similar problems in the CS limit in both $\mathrm{U}(1)$ and $\mathrm{SU}\left(N_{c}\right)$ models with fermions.

\section{Acknowledgments}

I am grateful to A. P. Bakulev, D.J. Broadhurst, S.J. Brodsky, S. V. Mikhailov, D. Mueller, M.V. Polyakov, N. Stefanis, K.V. Stepanyntz, O.V. Teryaev and D.V. Shirkov for their in- 
terest in subjects related to this work. I also wish to thank S.A. Larin for useful discussions in the process of his participation at the preliminary stage of these studies, which resulted in part in his personal work of ref. [37]. The work was supported by Grants RFBR No. 11-01-00182, RFBR No. 11-02-00112 and is done within the scientific program of the the Grant NS-2835.2014.2.

Open Access. This article is distributed under the terms of the Creative Commons Attribution License (CC-BY 4.0), which permits any use, distribution and reproduction in any medium, provided the original author(s) and source are credited.

\section{References}

[1] E.J. Schreier, Conformal symmetry and three-point functions, Phys. Rev. D 3 (1971) 980 [INSPIRE].

[2] G.T. Gabadadze and A.L. Kataev, On connection between coefficient functions for deep inelastic and annihilation processes, JETP Lett. 61 (1995) 448 [Pisma Zh. Eksp. Teor. Fiz. 61 (1995) 439] [hep-ph/9502384] [INSPIRE].

[3] J. Erlich and D.Z. Freedman, Conformal symmetry and the chiral anomaly, Phys. Rev. D 55 (1997) 6522 [hep-th/9611133] [INSPIRE].

[4] D.Z. Freedman, K. Johnson and J.I. Latorre, Differential regularization and renormalization: a new method of calculation in quantum field theory, Nucl. Phys. B 371 (1992) 353 [InSPIRE].

[5] G. 't Hooft and M.J.G. Veltman, Regularization and renormalization of gauge fields, Nucl. Phys. B 44 (1972) 189 [INSPIRE].

[6] C.G. Bollini and J.J. Giambiagi, Dimensional renormalization: the number of dimensions as a regularizing parameter, Nuovo Cim. B 12 (1972) 20 [INSPIRE].

[7] C.G. Bollini and J.J. Giambiagi, Lowest order divergent graphs in nu-dimensional space, Phys. Lett. B 40 (1972) 566 [InSPIRE].

[8] J.F. Ashmore, A method of gauge invariant regularization, Lett. Nuovo Cim. 4 (1972) 289 [INSPIRE].

[9] G.M. Cicuta and E. Montaldi, Analytic renormalization via continuous space dimension, Lett. Nuovo Cim. 4 (1972) 329 [INSPIRE].

[10] W.A. Bardeen, A. J. Buras, D.W. Duke and T. Muta, Deep inelastic scattering beyond the leading order in asymptotically free gauge theories, Phys. Rev. D 18 (1978) 3988 [INSPIRE].

[11] A.L. Kataev and M.D. Vardiashvili, Scheme dependence of the perturbative series for a physical quantity in the $g \phi^{4}$ theory, Phys. Lett. B 221 (1989) 377 [Erratum ibid. B 241 (1990) 644] [INSPIRE].

[12] F. Jegerlehner and O.V. Tarasov, Explicit results for the anomalous three point function and non-renormalization theorems, Phys. Lett. B 639 (2006) 299 [hep-ph/0510308] [INSPIRE].

[13] V.A. Smirnov, Differential renormalization and dimensional regularization, Nucl. Phys. B 427 (1994) 325 [INSPIRE].

[14] R.J. Crewther, S.S. Shei and T.M. Yan, Axial-vector currents in finite theories of quantum electrodynamics, Phys. Rev. D 8 (1973) 3396 [INSPIRE]. 
[15] K. Johnson, R. Willey and M. Baker, Vacuum polarization in quantum electrodynamics, Phys. Rev. 163 (1967) 1699 [INSPIRE].

[16] S.L. Adler, Short distance behavior of quantum electrodynamics and a eigenvalue condition for alpha, Phys. Rev. D 5 (1972) 3021 [INSPIRE].

[17] K. Johnson and M. Baker, Some speculations on high-energy quantum electrodynamics, Phys. Rev. D 8 (1973) 1110 [INSPIRE].

[18] A.A. Vladimirov and D.V. Shirkov, The renormalization group and ultraviolet asymptotics, Sov. Phys. Usp. 22 (1979) 860 [Usp. Fiz. Nauk 129 (1979) 407] [INSPIRE].

[19] S.G. Gorishny, A.L. Kataev, S.A. Larin and L.R. Surguladze, The analytical four loop corrections to the $Q E D \beta$-function in the $M S$ scheme and to the QED $\psi$-function: total reevaluation, Phys. Lett. B 256 (1991) 81 [INSPIRE].

[20] A.L. Kataev, Riemann $\zeta(3)$-terms in perturbative QED series, conformal symmetry and the analogies with structures of multiloop effects in $N=4$ supersymmetric Yang-Mills theory, Phys. Lett. B 691 (2010) 82 [arXiv: 1005.2058] [inSPIRE].

[21] A.L. Kataev, The generalized crewther relation: the peculiar aspects of the analytical perturbative QCD calculations, in Minneapolis 1996, Continuous advances in QCD, M.I. Polikarpov ed., World Scientific, Singapore (1996), hep-ph/9607426 [INSPIRE].

[22] A.L. Kataev, Conformal symmetry based relation between Bjorken and Ellis-Jaffe sum rules, DESY-PROC-2012-02 (2012) [arXiv: 1207. 1808] [INSPIRE].

[23] R.J. Crewther, Nonperturbative evaluation of the anomalies in low-energy theorems, Phys. Rev. Lett. 28 (1972) 1421 [INSPIRE].

[24] S.L. Adler, C.G. Callan Jr., D.J. Gross and R. Jackiw, Constraints on anomalies, Phys. Rev. D 6 (1972) 2982 [INSPIRE].

[25] D.J. Broadhurst and A.L. Kataev, Connections between deep inelastic and annihilation processes at next to next-to-leading order and beyond, Phys. Lett. B 315 (1993) 179 [hep-ph/9308274] [INSPIRE].

[26] S.A. Larin and J.A.M. Vermaseren, The $\alpha_{s}^{3}$ corrections to the Bjorken sum rule for polarized electroproduction and to the Gross-Llewellyn Smith sum rule, Phys. Lett. B 259 (1991) 345 [INSPIRE].

[27] S.G. Gorishny, A.L. Kataev and S.A. Larin, The $O\left(\alpha_{s}^{3}\right)$ corrections to $\sigma_{\text {tot }}\left(e^{+} e^{-} \rightarrow\right.$ hadrons $)$ and $\Gamma\left(\tau^{-} \rightarrow \tau\right.$-neutrino + hadrons $)$ in QCD, Phys. Lett. B 259 (1991) 144 [INSPIRE].

[28] L.R. Surguladze and M.A. Samuel, Total hadronic cross-section in $e^{+} e^{-}$annihilation at the four loop level of perturbative QCD, Phys. Rev. Lett. 66 (1991) 560 [Erratum ibid. 66 (1991) 2416] [INSPIRE].

[29] S.G. Gorishny, A.L. Kataev and S.A. Larin, Correction $O\left(\alpha_{s}^{3} \rightarrow \sigma_{\text {tot }}\left(e^{+} e^{-} \rightarrow\right.\right.$ hadrons $)$ in quantum chromodynamics, JETP Lett. 53 (1991) 127 [Pisma Zh. Eksp. Teor. Fiz. 53 (1991) 121] [INSPIRE].

[30] K.G. Chetyrkin, Corrections of order $\alpha_{s}^{3}$ to $R_{\text {had }}$ in $p Q C D$ with light gluinos, Phys. Lett. B 391 (1997) 402 [hep-ph/9608480] [INSPIRE].

[31] P.A. Baikov, K.G. Chetyrkin and J.H. Kuhn, Adler function, Bjorken sum rule and the Crewther relation to order $\alpha_{s}^{4}$ in a general gauge theory, Phys. Rev. Lett. 104 (2010) 132004 [arXiv: 1001.3606] [INSPIRE]. 
[32] P.A. Baikov, K.G. Chetyrkin, J.H. Kuhn and J. Rittinger, Adler function, sum rules and Crewther relation of order $O\left(\alpha_{s}^{4}\right)$ : the singlet case, Phys. Lett. B $714(2012) 62$ [arXiv: 1206.1288] [INSPIRE].

[33] O.V. Tarasov, A.A. Vladimirov and A.Y. Zharkov, The Gell-Mann-Low function of QCD in the three loop approximation, Phys. Lett. B 93 (1980) 429 [INSPIRE].

[34] S.A. Larin and J.A.M. Vermaseren, The three loop $Q C D \beta$-function and anomalous dimensions, Phys. Lett. B 303 (1993) 334 [hep-ph/9302208] [INSPIRE].

[35] R.J. Crewther, Relating inclusive $e^{+} e^{-}$annihilation to electroproduction sum rules in quantum chromodynamics, Phys. Lett. B 397 (1997) 137 [hep-ph/9701321] [INSPIRE].

[36] V.M. Braun, G.P. Korchemsky and D. Mueller, The uses of conformal symmetry in QCD, Prog. Part. Nucl. Phys. 51 (2003) 311 [hep-ph/0306057] [InSPIRE].

[37] S.A. Larin, The singlet contribution to the Bjorken sum rule for polarized deep inelastic scattering, Phys. Lett. B 723 (2013) 348 [arXiv:1303.4021] [INSPIRE].

[38] A.L. Kataev, The analytical singlet $\alpha_{s}^{4} Q C D$ contributions into the $e^{+} e^{-}$-annihilation Adler function and the generalized Crewther relations, JETP Lett. 94 (2011) 789 [Pisma Zh. Eksp. Teor. Fiz. 94 (2011) 867] [arXiv: 1108.2898] [INSPIRE].

[39] E. Gardi and M. Karliner, Relations between observables and the infrared fixed point in QCD, Nucl. Phys. B 529 (1998) 383 [hep-ph/9802218] [INSPIRE].

[40] R. Shrock, Study of scheme transformations to remove higher-loop terms in the $\beta$ function of a gauge theory, Phys. Rev. D 88 (2013) 036003 [arXiv: 1305.6524] [INSPIRE].

[41] R. Armillis, A. Monin and M. Shaposhnikov, Spontaneously broken conformal symmetry: dealing with the trace anomaly, JHEP 10 (2013) 030 [arXiv:1302.5619] [INSPIRE].

[42] A.V. Efremov and O.V. Teryaev, Spin structure of the nucleon and triangle anomaly, JINR-E2-88-287 (1988).

[43] G. Altarelli and G.G. Ross, The anomalous gluon contribution to polarized leptoproduction, Phys. Lett. B 212 (1988) 391 [INSPIRE].

[44] E.B. Zijlstra and W.L. van Neerven, Order $\alpha_{s}^{2}$ corrections to the polarized structure function $g_{1}\left(x, Q^{2}\right)$, Nucl. Phys. B 417 (1994) 61 [Erratum ibid. B 426 (1994) 245] [Erratum ibid. B $\mathbf{7 7 3}$ (2007) 105] [INSPIRE].

[45] S.A. Larin, T. van Ritbergen and J.A.M. Vermaseren, The $\alpha_{s}^{3}$ approximation of quantum chromodynamics to the Ellis-Jaffe sum rule, Phys. Lett. B 404 (1997) 153 [hep-ph/9702435] [INSPIRE].

[46] S.A. Larin, The next-to-leading QCD approximation to the Ellis-Jaffe sum rule, Phys. Lett. B 334 (1994) 192 [hep-ph/9403383] [INSPIRE].

[47] P. Cvitanovic, Group theory for Feynman diagrams in non-abelian gauge theories, Phys. Rev. D 14 (1976) 1536 [INSPIRE].

[48] T. van Ritbergen, J.A.M. Vermaseren and S.A. Larin, The four loop $\beta$-function in quantum chromodynamics, Phys. Lett. B 400 (1997) 379 [hep-ph/9701390] [INSPIRE].

[49] T. van Ritbergen, A.N. Schellekens and J.A.M. Vermaseren, Group theory factors for Feynman diagrams, Int. J. Mod. Phys. A 14 (1999) 41 [hep-ph/9802376] [INSPIRE]. 
[50] I. Jack, D.T. Jones, P. Kant and L. Mihaila, The four-loop DRED gauge $\beta$-function and fermion mass anomalous dimension for general gauge groups, JHEP 09 (2007) 058 [arXiv: 0707.3055] [INSPIRE].

[51] D.J. Broadhurst, Four loop Dyson-Schwinger-Johnson anatomy, Phys. Lett. B 466 (1999) 319 [hep-ph/9909336] [INSPIRE].

[52] P.A. Baikov, K.G. Chetyrkin and J.H. Kuhn, Massless propagators: applications in QCD and QED, PoS (RAD COR 2007) 023 [arXiv:0810.4048] [INSPIRE].

[53] A.L. Kataev, Is it possible to check urgently the 5-loop analytical results for the $e^{+} e^{-}$-annihilation Adler function?, Phys. Lett. B 668 (2008) 350 [arXiv:0808.3121] [INSPIRE].

[54] S.G. Gorishny and S.A. Larin, Coefficient functions of asymptotic operator expansions in minimal subtraction scheme, Nucl. Phys. B 283 (1987) 452 [InSPIRE].

[55] S.G. Gorishny and S.A. Larin, QCD corrections to the parton model rules for structure functions of deep inelastic scattering, Phys. Lett. B 172 (1986) 109 [INSPIRE].

[56] G.T. Gabadadze and A.A. Pivovarov, The Adler-Bardeen theorem for the axial Abelian anomaly in two-dimensional and four-dimensional theories, Phys. Atom. Nucl. 56 (1993) 565 [INSPIRE].

[57] S.V. Mikhailov, Generalization of BLM procedure and its scales in any order of $p Q C D$ : a practical approach, JHEP 06 (2007) 009 [hep-ph/0411397] [INSPIRE].

[58] A.L. Kataev and S.V. Mikhailov, New perturbation theory representation of the conformal symmetry breaking effects in gauge quantum field theory models, Theor. Math. Phys. 170 (2012) 139 [Teor. Mat. Fiz. 170 (2012) 174] [arXiv:1011.5248] [INSPIRE].

[59] S.J. Brodsky, G.P. Lepage and P.B. Mackenzie, On the elimination of scale ambiguities in perturbative quantum chromodynamics, Phys. Rev. D 28 (1983) 228 [INSPIRE].

[60] A.L. Kataev, QCD scale scheme fixing prescriptions at the next next-to-leading level, in the proceedings of the $27^{\text {th }}$ Recontre de Moriond " $Q C D$ and High-Energy Hadronic Interactions", March 22-28 Les-Arcs, France (1992).

[61] G. Grunberg and A.L. Kataev, On some possible extensions of the Brodsky-Lepage-MacKenzie approach beyond the next-to-leading order, Phys. Lett. B 279 (1992) 352 [INSPIRE].

[62] M. Mojaza, S.J. Brodsky and X.-G. Wu, A systematic all-orders method to eliminate renormalization-scale and scheme ambiguities in PQCD, Phys. Rev. Lett. 110 (2013) 192001 [arXiv: 1212.0049] [INSPIRE].

[63] X.-G. Wu, S.J. Brodsky and M. Mojaza, The renormalization scale-setting problem in QCD, Prog. Part. Nucl. Phys. 72 (2013) 44 [arXiv:1302.0599] [INSPIRE].

[64] S.J. Brodsky, M. Mojaza and X.-G. Wu, Systematic scale-setting to all orders: the principle of maximum conformality and commensurate scale relations, Phys. Rev. D 89 (2014) 014027 [arXiv: 1304.4631] [INSPIRE].

[65] J. Mondejar and K. Melnikov, The VVA correlator at three loops in perturbative QCD, Phys. Lett. B 718 (2013) 1364 [arXiv: 1210.0812] [INSPIRE]. 